ORIGINAL ARTICLE

\title{
Inspiratory resistive loading improves cycling capacity: a placebo controlled trial
}

\section{A D Gething, M Williams, B Davies}

Br J Sports Med 2004;38:730-736. doi: 10.1136/bjsm.2003.007518

See end of article for authors' affiliations

\section{Correspondence to} Mr Gething, School of Applied Sciences, University of Glamorgan, Llantwit Road, Cardiff CF37 1DL, Wales, UK adgething@btopenworld. com

Accepted 6 October 2003
Background: Respiratory muscle training has been shown to improve both its strength and endurance. The effect of these improvements on whole-body exercise performance remains controversial.

Objective: To assess the effect of a 10 week inspiratory resistive loading (IRL) intervention on respiratory muscle performance and whole-body exercise endurance.

Methods: Fifteen apparently healthy subjects (10 men, 5 women) were randomly allocated to one of three groups. One group underwent IRL set at $80 \%$ of maximum inspiratory pressure with ever decreasing work/rest ratios until task failure, for three days a week for 10 weeks (IRL group). A second placebo group performed the same training procedure but with a minimal resistance (PLA group). IRL and placebo training were performed at rest. The remaining five control subjects performed no IRL during the 10 week study period (CON group). Cycling endurance capacity at $75 \% \dot{\mathrm{V}}_{2}$ peak was measured before and after the intervention.

Results: After the 10 week IRL intervention, respiratory muscle strength (maximum inspiratory pressure) and endurance (sum of sustained maximum inspiratory pressure) had significantly improved (by $34 \%$ and $38 \%$ respectively). An increase in diaphragm thickness was also observed. These improvements translated into a $36 \%$ increase in cycling time to exhaustion at $75 \% \dot{V}_{\mathrm{O}_{2}}$ peak. During cycling trials, heart rate, ventilation, and rating of perceived exertion were attenuated in the IRL group. No changes were observed for the PLA or CON group either in the time to exhaustion or cardiorespiratory response to the same intensity of exercise.

Conclusion: Ten weeks of IRL attenuated the heart rate, ventilatory, and perceptual response to constant workload exercise, and improved the cycling time to exhaustion. Familiarisation was not a factor and the placebo effect was minimal.
S ince it has been possible to specifically train the respiratory muscles to improve strength and/or endurance, ${ }^{1}$ the effect of doing so on whole-body exercise performance has stimulated many studies. ${ }^{2}$ Despite this, results remain equivocal, with some studies reporting improvements in exercise capacity ${ }^{3-10}$ and others reporting no significant effect. ${ }^{11-16}$ The different methods of respiratory training and performance outcomes used have made it difficult to determine when respiratory muscle training (RMT) will result in improved performance. Most RMT studies have used one of two modes of training: (a) voluntary isocapnic hyperpnoea (VIH) to improve respiratory muscle endurance; $(b)$ inspiratory resistive loading (IRL) to improve respiratory muscle strength. ${ }^{2}$ IRL can be achieved with either pressure threshold or flow resistive devices. A pressure threshold device requires subjects to produce a negative pressure sufficient to overcome a threshold load. Threshold loading in this manner allows variable loading at a quantifiable intensity by providing near flow independent resistance to inspiration and has been used successfully in several recent studies. ${ }^{6710}$ In contrast, inspiratory flow resistive loading requires subjects to inspire through a variable diameter orifice so that, for a given flow rate, the smaller the orifice the greater is the resistive load. This method has received little specific attention.

Most RMT studies have lacked an effective placebo ${ }^{34}{ }^{16}$ or a control group ${ }^{6-8}$ or have not used repeated trials for constant workload performance outcomes to account for familiarisation. ${ }^{17}$ Therefore, despite a positive outcome, the extent to which the improvement is due to an actual training effect is difficult to ascertain. More recent studies have included a placebo group while assessing RMT and performance. ${ }^{6} 1017$
These studies have shown an improvement in performance after an RMT intervention, but, whereas Sonetti et al ${ }^{17}$ reported an improvement in the placebo group equal to that of the training group, in the study of Volianitis et al, ${ }^{10}$ the improvement in the trained group was significantly greater than the improvement in the placebo group. However, in the latter study, the improvements in the placebo group were expected to have resulted from whole-body training (subjects were in the preseason phase of training) and probably not a placebo effect. Finally, Romer $e t a l^{6}$ showed no improvement in the placebo group. The extent of the placebo effect on exercise performance with this type of intervention is difficult to ascertain because all studies lacked a control group-that is, a group who receive no training.

The following study was designed to assess the effect of a 10 week IRL intervention on respiratory muscle performance and whole-body exercise endurance. Familiarisation (by using repeat trials) and the placebo effect (by using a placebo and a control group) were also considered. The study also provides data on a range of physiological and perceptual variables under identical conditions before and after the intervention. We hypothesised that IRL would significantly improve cycling endurance capacity over and above any improvements observed in the placebo and control groups.

\footnotetext{
Abbreviations: IRL, inspiratory resistive loading; MEP, maximum expiratory pressure; MIP, maximum inspiratory pressure; RMT, respiratory muscle training; RPE, rating of perceived exertion; TIRE, the test of incremental respiratory endurance; SMIP, sustained maximum inspiratory pressure; $\mathrm{VIH}$, voluntary isocapnic hyperpnoea; $\mathrm{VO}_{2}$ peak, peak oxygen consumption; Wmax, maximal power output
} 


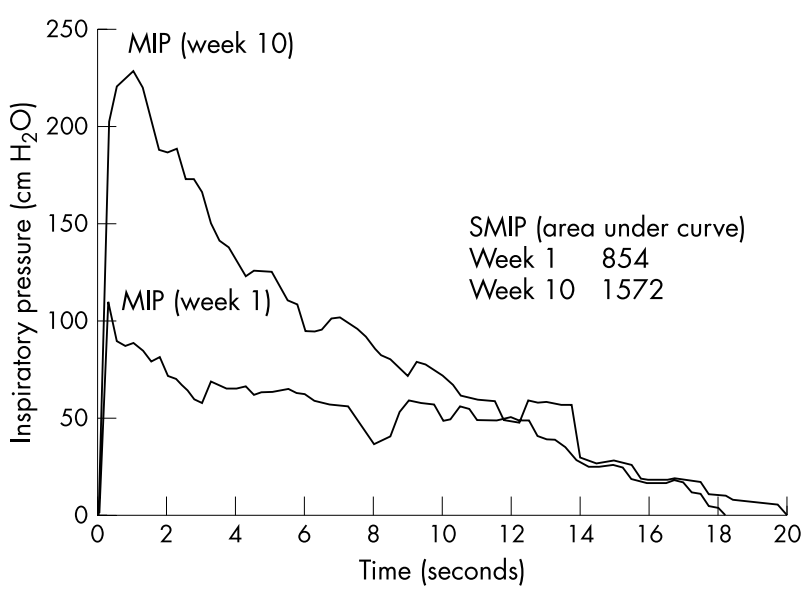

Figure 1 Test of incremental respiratory endurance (TIRE) templates at week 1 and 10 of inspiratory resistive loading for one subject. MIP, Maximum inspiratory pressure; SMIP, sustained maximum inspiratory pressure.

\section{METHODS}

Fifteen apparently healthy subjects ( 10 men, 5 women) who exercised regularly were recruited. Written informed consent was obtained from each subject, and the ethics committee of the University of Glamorgan approved all procedures. The physical characteristics of the subjects were (values are mean (SD)): age 22.7 (2.3) years; stature 175 (8.6) cm; body mass 75.8 (9.6) kg; peak oxygen consumption ( $\left(\dot{\mathrm{V}}_{2}\right.$ peak) 3.18 (0.69) litres/min; maximal power output (Wmax) 287 (64) W.

\section{Procedure}

Subjects presented to the laboratory after a 12 hour overnight fast, having consumed $500 \mathrm{ml}$ water two hours before arrival to ensure that they were euhydrated, as confirmed during a pilot study. Subjects also provided a 48 hour dietary recall on the initial visit to the laboratory, and were given a copy of this diet to follow for the 48 hours before the next visit, and every time thereafter.

Each subject visited the laboratory four times before the intervention and three times after. The first visit comprised a spirometric assessment, maximum mouth pressures, and a submaximal cycling test to establish their oxygen consumption $v$ workload relation. On the same day, an incremental test to exhaustion was used to establish $\dot{\mathrm{V}}_{2}$ peak. One week later, subjects completed a cycling trial to exhaustion at a resistance prescribed to elicit $75 \% \dot{\mathrm{V}}_{2}$ peak $\left(\mathrm{Tlim}_{75}\right)$. Subjects repeated this trial at the same resistance on the two remaining visits to the laboratory, again separated by one

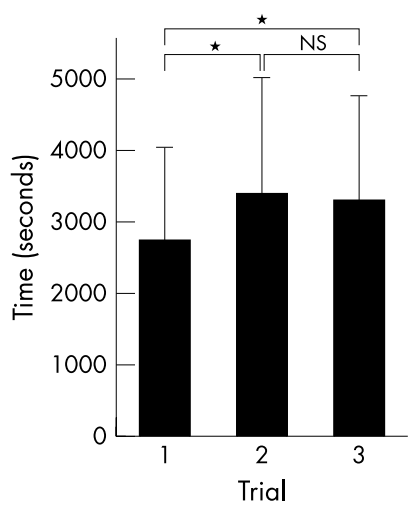

Figure 2 Changes in cycling time to exhaustion at $75 \% \mathrm{~V}_{2}$ peak as a result of familiarisation $(n=15)$. * Significantly different from value before the test $(p<0.05)$. week to avoid physiological adaptations. Subjects then received a 10 week intervention of IRL, placebo (PLA), or no IRL training (CON) to which they were randomly allocated. The effects of the interventions were evaluated using the same battery of tests after the respective interventions over a three week period.

\section{Lung function tests}

Forced expiratory volume in one second $\left(\mathrm{FEV}_{1}\right)$, forced vital capacity (FVC), $\mathrm{FEV}_{\mathrm{l}} / \mathrm{FVC}$, and peak expiratory flow (PEF) were determined using a computerised spirometry system (Spirosense Spirometry System; Burdick Inc, Milton, Wisconsin, USA). The highest value from each set of three repeated measurements for each test was used for subsequent analysis. ${ }^{18}$

Mouth pressures (maximum inspiratory pressure (MIP) and maximum expiratory pressure (MEP)) were measured with a portable hand held mouth pressure meter (Micro Medical Ltd, Rochester, Kent, UK). Subjects were strongly urged to make maximum inspiratory (Mueller manoeuvre) and expiratory (Valsalva manoeuvre) efforts at or near residual volume and total lung capacity respectively. The maximum of three manoeuvres was recorded. ${ }^{19}$

\section{Exercise testing}

All exercise tests were conducted on the same cycle ergometer (Monark 864, Varberg, Sweden).

The relation between workload or power output and oxygen consumption was calculated by selecting four submaximal workloads that would elicit a heart rate ranging from 120 to 180 beats/min and measuring oxygen consumption using a computerised $\mathrm{O}_{2}$ breath by breath analysis system (Medgraphics CPX/D, St Paul, Minnesota, USA).

Subjects next performed an incremental test to exhaustion starting at a power output of $60 \mathrm{~W}$ and increasing every minute by $24 \mathrm{~W}$ until the criteria for $\dot{\mathrm{V}}_{2}$ peak were reachedthat is, rating of perceived exertion (RPE) of 20, volitional exhaustion, respiratory exchange ratio $>1.1$, heart rate equal to or greater than maximum $(220-$ age $)$.

For each subject, $75 \% \dot{\mathrm{V}}_{2}$ peak was ascertained from the regression of workload $v$ oxygen consumption to determine the resistance required to elicit this oxygen consumption. Each test continued until volitional fatigue, defined as failure to maintain cadence $\geqslant 60 \mathrm{rpm}$. During the trial, measurements were made of oxygen consumption $\left(\dot{\mathrm{V}}_{2}\right)$, ventilation, heart rate, respiratory exchange ratio, and RPE.

Subjects performed the trial three times at baseline, with data from the first trial excluded to allow for familiarisation. The best time of the second and third trials was taken as $\mathrm{Tlim}_{75}$ in an attempt to account for some of the day to day variation common to this type of trial. After the intervention, the subjects performed just two trials, as they should then have been familiarised with the test. Again Tlim $_{75}$ was taken as the best of the two trials. More than 24 hours was allowed between completion of the last IRL session and re-evaluation of exercise capacity to provide adequate time for respiratory muscle fatigue to subside. ${ }^{20}$

\section{Inspiratory muscle training}

Both IRL and placebo training were conducted three days a week for 10 weeks (30 sessions). All training, both IRL and placebo, was supervised by the same investigator (AG) to ensure $100 \%$ adherence. Subjects continued with their regular exercise training programmes and were required to keep a diary throughout the study in which both the frequency and duration of training were recorded.

The IRL device is flow resistive, with subjects having to breathe through a $2 \mathrm{~mm}$ leak, present to prevent glottal pressure. A maximum flow was set during the inspiratory 
Table 1 Lung function at baseline

\begin{tabular}{llll}
\hline Variable & IRL & PLA & CON \\
\hline FEV $_{1}$ (litres) & $3.7(0.7)$ & $3.7(1.0)$ & $3.9(0.80$ \\
FVC (litres) & $(103(12))$ & $(93(8))$ & $(94(10))$ \\
FEV $_{1} / \mathrm{FVC}$ ratio & $4.4(0.6)$ & $4.6(1.1)$ & $4.9(0.9)$ \\
PEF (litres/s) & $(106(10))$ & $(99(4))$ & $(98(9))$ \\
& $0.84(0.06)$ & $0.80(0.06)$ & $0.81(0.08)$ \\
& $(97(8))$ & $(95(10))$ & $(96(10))$ \\
& $10.5(1.8)$ & $8.7(1.6)$ & $8.2(2.4)$ \\
\hline
\end{tabular}

Values are mean $(S D)(n=5)$. Values in parentheses are the percentage of predicted value based on age, height, and sex. ${ }^{18}$ Values obtained after training are not shown.

IRL, Inspiratory resistive loading; PLA, placebo; $C O N$, control; $F_{1}$, forced expiratory volume in one second; FVC, forced vital capacity; PEF, peak expiratory flow.

effort proportional to the pressure achieved. The measured resistance (pressure/flow) was about $270 \mathrm{~cm} \mathrm{H}_{2} \mathrm{O} /$ litre/s. Placebo subjects used the same breathing device with a different mouthpiece which had a greatly reduced flow resistance (a leak of $30 \mathrm{~mm}$, resistance about $10 \mathrm{~cm} \mathrm{H}_{2} \mathrm{O}$ / litre/s).

IRL was undertaken using the test of incremental respiratory endurance (TIRE) system, which has previously been described. ${ }^{21}$ Briefly, during the TIRE, respiratory work is fixed in direct relation to individual capacity by establishing the subjects' sustained maximum inspiratory pressure (SMIP). This is accomplished by encouraging the subject to inspire from residual volume to total lung capacity, through the full lung volume. Pressure is measured throughout the manoeuvre, with SMIP taken as the area under the curve (fig 1). Computer generated targets are presented to the subject, set at $80 \%$ of maximum, across the functional range of volume, and the frequency at which the templates are presented increases throughout the test. The initial rest period between inspirations is 60 seconds, and this is reduced every six manoeuvres to $45,30,15,10$, and finally 5 seconds. If at any point during the TIRE, the subject fails to achieve at least $90 \%$ of this reduced template, the test is over. At each training session, SMIPs are reassessed so that the work performed during the TIRE on that day is based on the new maximal effort.

\section{Statistical analysis}

Shapiro-Wilks tests were applied to each dependent variable to confirm distribution normality. Mixed between-within

Table 2 Effect of IRL on respiratory muscle function

\begin{tabular}{|c|c|c|c|c|c|c|}
\hline \multirow[b]{2}{*}{ Variable } & \multicolumn{2}{|l|}{ IRL } & \multicolumn{2}{|l|}{ PLA } & \multicolumn{2}{|l|}{ CON } \\
\hline & Before & After & Before & After & Before & After \\
\hline MIP & 134 & 180 & 136 & 140.2 & 127 & 128 \\
\hline$\left(\mathrm{cm} \mathrm{H}_{2} \mathrm{O}\right)$ & (35) & $(39)^{*}$ & (26) & (29) & (40) & (39) \\
\hline MEP & 138 & 144 & 126 & 126 & 117 & 126 \\
\hline$\left(\mathrm{cm} \mathrm{H}{ }_{2} \mathrm{O}\right)$ & (26) & (26) & (40) & (8) & (21) & (32) \\
\hline SMIP & 987 & 1303 & $\sim$ & $\sim$ & 897 & 915 \\
\hline (PTU) & (144) & $(212)^{*}$ & & & (231) & (219) \\
\hline$\Sigma S M I P$ & 29.6 & 40.7 & $\sim$ & $\sim$ & 23.9 & 24.9 \\
\hline (PTU) & (3.1) & $(5.8)^{*}$ & & & (1.1) & (2.9) \\
\hline Time & 13.6 & 17.4 & $\sim$ & $\sim$ & 15.8 & 15.8 \\
\hline (seconds) & (3.0) & $(1.6)^{*}$ & & & (3.6) & (4.4) \\
\hline
\end{tabular}

Values are mean (SD) ( $n=5)$.

*Significantly different change from baseline $(p<0.05)$.

IRL, Inspiratory resistive loading; PLA, placebo; CON, control; MIP, maximum inspiratory pressure; $M E P$, maximum expiratory pressure; SMIP, sustained maximum inspiratory pressure; $\Sigma$ SMIP, sum of SMIP; PTU, pressure time unit; Time, duration of inspiration. These variables were not measured in the placebo group because performing the test of incremental respiratory endurance with normal resistance would unblind the study. analyses of variance were used to test for between-group effects of the treatment (IRL, PLA, CON) and within-group effects of the intervention (before and after treatment). To determine where significant differences existed between pairs of mean values, Student's paired sample $t$ tests were used with a Bonferroni correction factor. Specifically, this analysis assessed the changes in mean values over time in each of the two types of performance tests, using peak power and $\dot{\mathrm{V}}_{2}$ peak for the incremental test, and time to exhaustion in the Tlim $_{75}$ trials. Changes in cardiorespiratory responses and RPE measured at fixed time points during Tlim 75 were examined in the same manner. To examine the effect of familiarisation on the first three trials, we used multiple Student's paired samples $t$ test with a Bonferroni correction. Significance for all two tailed tests was established at an $\alpha$ level of $\mathrm{p}<0.05$, and data are expressed as mean (SD).

\section{RESULTS}

\section{Familiarisation}

To account for familiarisation in our study, we used repeated trials. Figure 2 shows the cycling time to exhaustion at $75 \%$ $\dot{\mathrm{V}}_{2}$ peak for all subjects (regardless of group) before the intervention. The times for both the second and the third trial are both significantly greater than the time of the initial trial

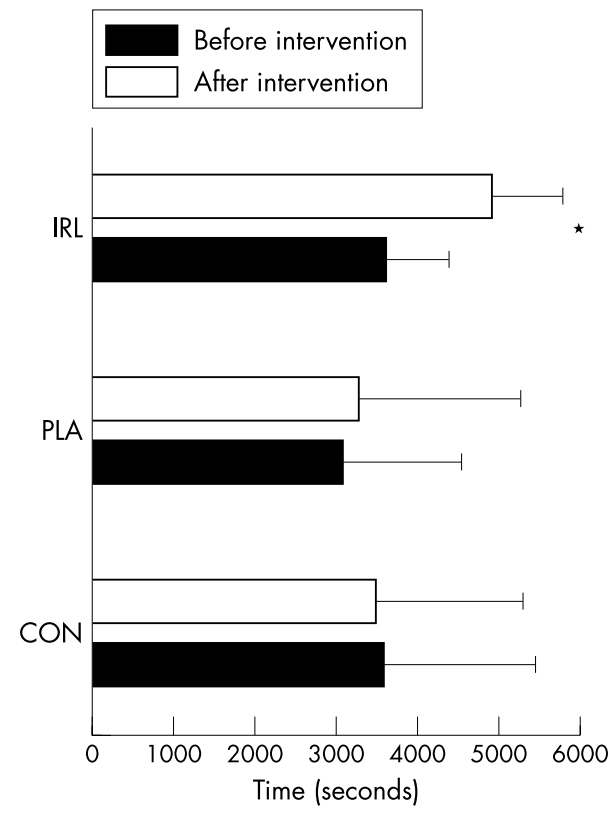

Figure 3 Effects of 10 weeks of inspiratory resistive loading (IRL), placebo (PLA), or no respiratory training (CON) on Tlim 75 before and after the intervention. All data are the best of the two trials. *Significantly different $(p<0.05)$. 

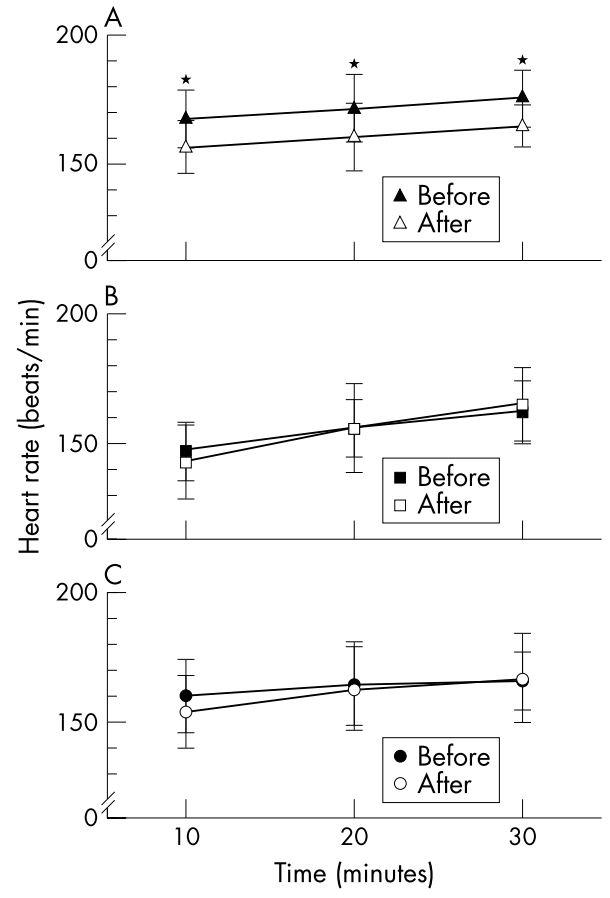

Figure 4 Effects of 10 weeks of inspiratory resistive loading $(A)$, placebo (B), or no respiratory training $(C)$ on heart rate during the Tlim 75 . Values are mean (SD). *Significantly different from value before the test $(p<0.05)$.

$(p<0.05)$. Importantly, there was no significant difference between the times of the second and third trials.

\section{Adherence to training}

For both groups that were expected to perform respiratory training-that is, both the PLA and IRL group-there was $100 \%$ adherence, with all 10 subjects completing 30 sessions during the 10 week period. In addition, supervision of the training, with appropriate encouragement ensured that subjects were fully motivated, producing a maximal training template, at the start of each training session.

\section{Whole-body training}

All subjects continued their regular exercise training programmes during the 10 week intervention period and were required to keep a diary of all physical activity. During this period, training did not differ between/within the three groups in terms of the frequency and duration of training, although no record of the intensity of training was recorded. In week 1 of the training intervention, the frequency of whole-body training was 4.4 (1.1), 4.8 (1.1), and 3.8 (1.5) sessions/week for the IRL, PLA, and CON groups respectively. Duration of training was 198 (91), 200 (119), and 181 (68) $\mathrm{min} /$ week respectively. In the final week of training, the frequency $(4.2(0.8), 4.0(1.0), 4.0$ (1.2) sessions/week) and duration (197 (59), 198 (120), and 179 (78) min/week respectively) remained the same.

\section{Lung function}

Table 1 shows lung function, as assessed by $\mathrm{FEV}_{1}, \mathrm{FVC}, \mathrm{FEV}_{1} /$ FVC ratio, and PEF, measured at baseline. All values are within normal limits, and values after interventions were unremarkable.

\section{Respiratory muscle function}

After the intervention, MIP (an indicator of inspiratory muscle strength), SMIP, sum of SMIP (both indicators of

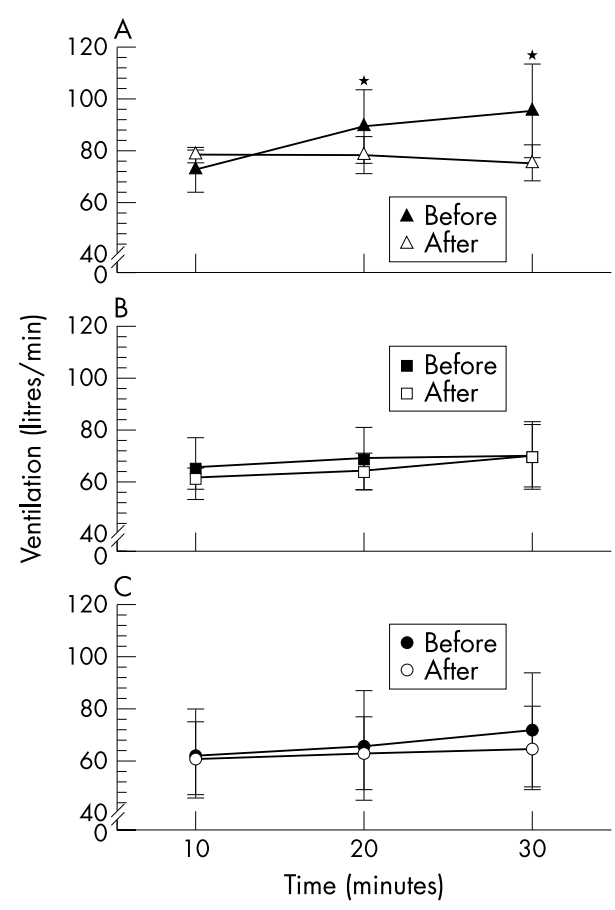

Figure 5 Effects of 10 weeks of inspiratory resistive loading (A), placebo (B), or no respiratory training $(C)$ on minute ventilation during the Tlim75. Values are mean (SD). *Significantly different from value before the test $(p<0.05)$.

inspiratory muscle endurance), and the duration of inspiration were all significantly increased compared with baseline in the IRL group (table 2). However, MEP was not improved after the intervention, demonstrating the specificity of the training to the inspiratory muscles. The control group, who performed one TIRE at baseline and again after the 10 week period, did not improve in any of the above variables.

\section{Incremental cycle test}

Maximum power output (Wmax) for the subjects did not differ between the IRL, PLA, and CON groups. Similarly, these values remained unchanged after the intervention for the three groups. $\dot{\mathrm{V}}_{2}$ peak remained unchanged in all three groups.

\section{Cycling endurance $\left(\mathrm{Tlim}_{75}\right)$}

Before the training programmes, cycling time to exhaustion did not differ between the three groups (IRL, PLA, CON). After the training intervention, $\operatorname{Tlim}_{75}$ had significantly improved by $36 \%$ in the IRL group $(\mathrm{p}<0.05)$, a change that was not apparent in either the PLA or CON groups (fig 3). In the IRL group, all five subjects improved their individual cycling time to exhaustion by a mean value of 1292 (607) seconds, whereas in the placebo group a mean increase of 202 (526) seconds was not significant. Of the controls, an overall decrease of -96 (157) seconds was also not significant.

Heart rate, ventilation, $\dot{\mathrm{V}}_{2}$, and RER were measured throughout the time to exhaustion trial. We compared these variables (before and after the intervention) at 10, 20, and 30 minutes into exercise. After the IRL intervention, heart rate was significantly decreased at 10, 20, and 30 minutes (fig 4), and ventilation was significantly decreased at 20 and 30 minutes (fig 5).

$\dot{\mathrm{V}}_{2}$, respiratory frequency, and RER were not significantly altered after the intervention for all three groups (table 3 ). 
Table 3 Respiratory frequency, $\dot{V}_{2}$, and respiratory exchange ratio during the cycling endurance trial

\begin{tabular}{|c|c|c|c|c|c|c|c|}
\hline \multirow[b]{2}{*}{ Variable } & \multirow{2}{*}{$\begin{array}{l}\text { Time } \\
\text { (min) }\end{array}$} & \multicolumn{2}{|l|}{ IRL } & \multicolumn{2}{|l|}{ PLA } & \multicolumn{2}{|l|}{ CON } \\
\hline & & Before & After & Before & After & Before & After \\
\hline \multirow[t]{3}{*}{$\mathrm{B}_{\mathrm{f}}$ (breaths/min) } & 10 & $34.2(4.0)$ & $34.6(2.2)$ & $34.2(4.2)$ & $33.3(4.9)$ & $31.6(5.4)$ & $32.8(6.3)$ \\
\hline & 20 & $38.0(4.4)$ & $37.2(3.1)$ & $37.8(5.2)$ & $36.5(2.1)$ & $33.2(6.1)$ & $32.8(4.7)$ \\
\hline & 30 & $44.2(10.6)$ & $39.4(3.3)$ & $44.0(8.0)$ & 45.3 (10.9) & $40.6(7.8)$ & $35.6(7.2)$ \\
\hline \multirow[t]{3}{*}{$\dot{\mathrm{V}}_{2}$ (litres/min) } & 10 & $2.6(0.3)$ & $2.7(0.2)$ & $1.9(0.4)$ & $1.9(0.2)$ & $2.2(0.7)$ & $2.2(0.4)$ \\
\hline & 20 & $2.6(0.3)$ & $2.7(0.3)$ & $1.9(0.4)$ & $2.0(0.3)$ & $2.2(0.8)$ & $2.2(0.5)$ \\
\hline & 30 & $2.6(0.5)$ & $2.8(0.2)$ & $1.8(0.5)$ & $2.0(0.4)$ & $2.2(0.8)$ & $2.2(0.4)$ \\
\hline \multirow[t]{3}{*}{ RER } & 10 & 1.04 (0.09) & $0.99(0.03)$ & $1.08(0.04)$ & $1.03(0.13)$ & $1.09(0.05)$ & $1.06(0.09)$ \\
\hline & 20 & $1.03(0.10)$ & $0.96(0.03)$ & $1.07(0.05)$ & $1.02(0.17)$ & $1.09(0.03)$ & $1.04(0.06)$ \\
\hline & 30 & $1.03(0.10)$ & $0.96(0.05)$ & $1.06(0.07)$ & $1.00(0.12)$ & $1.08(0.03)$ & $1.02(0.09)$ \\
\hline
\end{tabular}

Values are mean (SD) ( $\mathrm{n}=5)$.

IRL, Inspiratory resistive loading; PLA, placebo; $\mathrm{CON}$, control; $\mathrm{B}_{\mathrm{f}}$, respiratory frequency; $\dot{\mathrm{V}}_{2}$, oxygen consumption; RER, respiratory exchange ratio.

All subjects expressed their RPE at set time intervals throughout the trials using the Borg scale (fig 6). Substantial effects were observed for the IRL group during the trial, with RPE consistently lower for all subjects, but reaching significance only at the end of exercise (17.2 (1.1) and 15.4 $(0.89) ; \mathrm{p}<0.05)$ or when averaged across the whole trial ( 14.2 (1.3) before IRL and 12.9 (1.4) after IRL; $<<0.05$ ). There were no observed differences in the RPE for either the PLA or CON group (fig 6).

\section{DISCUSSION}

\section{Main findings of study}

The aim of this study was to assess the effect of a 10 week flow resistive IRL programme on the cycling time to exhaustion at an intensity prescribed to elicit $75 \%$ of $\dot{V} \mathrm{O}_{2}$ peak. A placebo and control group facilitated the effect of familiarisation and placebo. The main finding was that IRL attenuated the heart rate, ventilatory, and perceptual response to constant workload exercise, and improved the cycling time to exhaustion. Familiarisation was not a factor, and the placebo effect was minimal.

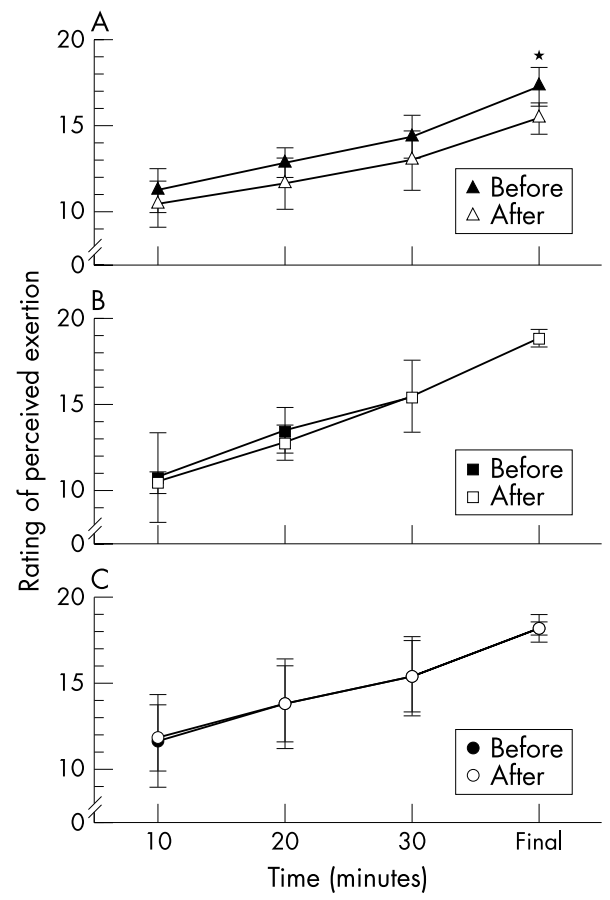

Figure 6 Effects of 10 weeks of inspiratory resistive loading (A), placebo $(B)$, or no respiratory training $(C)$ on rating of perceived exertion during the Tlim 75 . Values are mean (SD). *Significantly different from the value before the test $(p<0.05)$.

\section{Placebo effects}

The placebo intervention was designed to fulfil the criteria for a true placebo, as outlined by Ojaunen ${ }^{22}$ - that is, to be both inert and generate expectations, involvement, subjective utility, and be meaningful to the subjects. The placebo used in our study, which used a minimal exercise resistance, has been used in most studies of resistive training, ${ }^{6}{ }^{101323}$ but has been recently criticised for failing to activate the important placebo factors mentioned above. ${ }^{17}$ However, we feel that the assessment of our placebo effect, with all subjects exposed to the same respiratory protocol and biofeedback, was comprehensive

\section{Respiratory muscle function}

Inspiratory muscle strength, as measured by MIP, was improved in the IRL group but not in PLA or CON groups, whereas MEP was unchanged in all groups. The significant $34 \%$ increase in MIP shows the specificity of the training and is consistent with previous improvements reported after an IRL intervention, ${ }^{67101316}$ although Sonetti et al ${ }^{17}$ reported only an $8 \%$ improvement albeit after a shorter intervention (five week) using simultaneous extremes of training-that is, both IRL and VIH training-which may result in less than optimal muscular adaptation.

Previously, RMT performed at different lung volumes has had the greatest effect within the lung volume in which the training was prescribed. ${ }^{24}$ The method of training used in this study required subjects to inspire at $80 \%$ of their maximum through the full range of volume-that is, from residual volume to total lung capacity. Therefore an effect would be expected throughout the whole lung volume. This is reflected by the increase in the SMIP, determined by measuring pressure generation over a full inspiratory effort. A demonstrable increase throughout the inspiration in terms of the pressure generated was apparent for all subjects who received the IRL intervention (fig 1).

The inability to adequately fix workload or volume at which training occurred confounded earlier studies. ${ }^{2}$ Our methodology ensured accurate prescription of the workload, with reassessment of SMIP and resetting of the workload before each training session. In addition, biofeedback using an on screen training template was provided so that subjects could consistently perform IRL at $80 \%$ of the maximal inspiratory effort. Previously, this method has been shown to produce diaphragmatic fatigue, ${ }^{25}$ suggesting that the type of training used in our study provides an adequate stimulus to induce adaptation. There is scope for subjects to show a change in muscle contraction velocity verifiable by, for example, recording the time to peak pressure development before and after the intervention. However, because the technique used in this study is flow resistive, this is somewhat limited when compared with a pressure threshold device. An improvement in strength development rather than 
an increase in contraction velocity was the ultimate aim of the IRL intervention.

That respiratory muscle function can be improved after a specific RMT intervention is well established, ${ }^{2}$ but translation into an improvement in whole-body exercise performance is more controversial. ${ }^{11-13} 1617$ This controversy remains, with the different methods of respiratory training (VIH or IRL) and outcome measures (maximal exercise, endurance exercise, or performance) being the obvious confounding variables. Sonetti et $a l^{17}$ overcame this problem by combining the two types of training, but as pointed out by Romer et al, ${ }^{6}$ the concurrent strength and endurance training might have inhibited strength development and would explain the small $8 \%$ increase in MIP. Investigating and reviewing the results of resistive and hyperpnoea training separately may well provide insights into the mechanism behind RMT which is yet to be adequately explained.

\section{Maximal performance}

The improvements in respiratory muscle function observed in this study did not translate into an improvement in either $\dot{\mathrm{V}}_{2}$ peak or Wmax, which confirms previous findings on maximal exercise. ${ }^{61-13}$ The respiratory muscles do not limit $\dot{\mathrm{V}}_{2}$ peak, but rather are a part of it, just as much as any other muscles used during exercise. Lowering the cost of ventilation would increase the exercise intensity required to elicit $\dot{\mathrm{V}}_{2}$ peak, but $\dot{\mathrm{V}}_{2}$ peak would not be changed unless oxygen were more efficiently extracted by the locomotor muscles compared with those of the respiratory system. This has been shown to be the case using respiratory muscle unloading ${ }^{26}$ with a greater power output preserving $\dot{\mathrm{V}}_{2}$ peak, presumably because of the redistribution of blood flow. ${ }^{27}$ In our subjects, $\dot{\mathrm{V}}_{2}$ peak measures before and after IRL were unremarkable, and, although the difference is not significant, it is interesting that before IRL the maximum workload for the five subjects was $330 \mathrm{~W}$, and after it was $350 \mathrm{~W}$.

\section{Changes in $\operatorname{Tlim}_{75}$}

The observed improvements in respiratory muscle function represent a $36 \%$ increase in the cycling time to exhaustion at an intensity prescribed to elicit $75 \% \dot{\mathrm{VO}}_{2}$ peak. This is in line with previous studies, in which an increase in cycling time to exhaustion has been shown using both $\mathrm{VIH}^{3-5} 89$ and IRL techniques..$^{23}$ During the trial, a decrease in both heart rate and ventilation was also apparent. Previously, a decrease in minute ventilation after a VIH intervention has been reported, ${ }^{3}{ }^{4}$ but subsequent studies by the same group failed to substantiate these findings. ${ }^{589}$ A decrease in heart rate after an isocapnic hyperpnoea training intervention has been noted by a single study. ${ }^{28}$

It is unlikely that the improvements observed in this study were due to familiarisation, because of the rigorous nature of our testing protocol, which included averaging the results of two trials, both before and after intervention, and excluding the results of the initial trial (fig 2). Indeed, if familiarisation were a factor, despite our efforts, then we would have expected this to reveal itself by an improvement in $\mathrm{Tlim}_{75}$ in the control group, which was not manifest. The inclusion of both a placebo and control group in the study design allowed us to assess the magnitude of the placebo effect in our subjects (assuming familiarisation to be minimal). The improvements observed in our placebo group were not significant, and therefore not a major factor in the interpretation of these data.

We attempted to control for other possible explanations for the progress in Tlim $_{75}$ - for example, by providing our subjects with their previously recorded diets to follow in the 48 hour lead up to a trial. Although the subjects did not record exercise intensity-that is, heart rate-whole-body exercise training did not differ between or within the three groups, as determined by frequency and duration of training per week. Training intensity during the intervention is a potentially confounding variable, but, as all subjects understood the nature of the study, we do not consider it to be a major factor. For these reasons, we believe that the observed effects can be explained exclusively by the IRL intervention.

The experimental intervention in this study was therefore responsible for improvements in respiratory muscle function, which then, either directly or indirectly, resulted in an increase in the cycling time to exhaustion with a concomitant decrease in exercising heart rate, ventilation, and RPE during constant workload cycling.

\section{Mechanism of action}

Research into the mechanism behind respiratory training has focused on VIH. Several groups have systematically examined some of the possible mechanisms that could bring about the improvements in cycling endurance. To date, it has been shown that they are not due to a change in stroke volume ${ }^{5}$ or an increased oxygen supply as measured by blood gas concentrations. ${ }^{9}$ A decrease in blood lactate during endurance and after incremental exercise has been observed by some, ${ }^{78}$ but not all. ${ }^{17}$

A decrease in the RPE, as found in this study, is a candidate for the observed improvements in cycling endurance. Volianitis et $a l^{10}$ and Kellerman et $a^{29}$ reported a decrease in the perception of respiratory effort after a respiratory training intervention. In an outcome measure such as cycling time to exhaustion, which is by definition motivationally dependent, a decrease in the perception of exertion will probably have a profound effect on increasing $\operatorname{Tlim}_{75}$.

Another possibility to explain the effects of RMT is altered ventilatory efficiency. The reduction in ventilation observed in this study and others ${ }^{34}$ may contribute to the improvements in fixed work rate tests. The decrease in ventilation for a given workload will reduce the metabolic requirements of the respiratory muscles and result in diminished competition for blood flow requirements between the respiratory muscles and locomotor muscles. ${ }^{2}$ Blood flow redistribution is cited by some studies that have examined IRL, ${ }^{6}{ }^{10}$ although it has not been investigated directly. It follows a study in which reducing the work of breathing using a proportional assist ventilator during cycling exercise resulted in an increase in the blood flow to the legs. ${ }^{27}$ An average $50 \%$ reduction in the work of breathing results in a $5-7 \%$ increase in leg blood flow, and this translates into a $15 \%$ increase in endurance performance. However, it is very unlikely that the adaptations from a 10 week respiratory training protocol will decrease the work of breathing to anywhere near the 50\% decrease achieved using a ventilator. Therefore, although changes in leg blood flow may occur, they are likely to be very small and difficult to detect (C A Harms, personal communication). If a small reduction in the oxygen cost of breathing did occur after IRL (and this would explain the decrease in heart rate), it may be that our study lacked the statistical power to detect it.

A significant effect of an IRL intervention on exercising heart rate has not been previously reported, but has been shown after a VIH intervention, ${ }^{28}$ with an increase in stroke volume suggested as the potential mechanism. However, Markov et $a l^{5}$ has since reported no change in stroke volume after a VIH intervention. How the attenuation in heart rate observed in our study was brought about is not clear and requires further investigation, but the mechanism may be different from that involved in VIH.

Romer et $a l^{6}$ suggested that the mechanism behind the improvements in exercise performance was multifactorial, 


\section{What is already known on this topic}

IRL has been shown to improve exercise endurance capacity.

\section{What this study adds}

This study shows that these improvements are not due to the placebo effect or familiarisation. In addition, it provides data on a range of physiological and perceptual variables under identical exercise conditions before and after intervention.

with each of the above exerting some effect. We would agree with this statement, but would emphasise that the exact contribution of each remains unknown.

\section{CONCLUSION}

IRL using the TIRE device produced an increase in both the strength and endurance of the inspiratory muscles. These improvements resulted in an increase in cycling endurance at $75 \% \dot{\mathrm{V}}_{2}$ peak that was not apparent in either the placebo or control group. Familiarisation was kept to a minimum by the use of repeat trials, and the placebo effect was not a significant factor. This is the first study to adequately address the effect of IRL using both a placebo and control group. How these improvements influence actual competitive performance remains to be seen. The mechanisms behind the observed improvements have not yet received the required investigation, but continued studies in this area should provide the answer.

\section{ACKNOWLEDGEMENTS}

We acknowledge the contributions and assistance of $\mathrm{K}$ Chatham, A Davies, and L Passfield.

\section{Authors' affiliations}

A D Gething, M Williams, B Davies, School of Applied Sciences, University of Glamorgan, Cardiff, Wales, UK

Conflict of interest: none declared

\section{REFERENCES}

1 Leith DE, Bradley M. Ventilatory muscle strength and endurance training. J Appl Physiol 1976;41:508-16.

2 Sheel AW. Respiratory muscle training in healthy individuals: physiological rationale and implications for exercise performance. Sports Med 2002;32:567-81

3 Boutellier U, Buchel R, Kundert A, et al. The respiratory system as an exercise limiting factor in normal trained subjects. Eur J Appl Physiol Occup Physiol 1992:65:347-53.
4 Boutellier U, Piwko P. The respiratory system as an exercise limiting factor in normal sedentary subjects. Eur J Appl Physiol Occup Physiol 1992;64:145-52.

5 Markov G, Spengler CM, Knopfli-Lenzin C, et al. Respiratory muscle training increases cycling endurance without affecting cardiovascular responses to exercise. Eur J Appl Physiol 2001;85:233-9.

6 Romer LM, McConnell AK, Jones DA. Effects of inspiratory muscle training on time-trial performance in trained cyclists. J Sports Sci 2002;20:547-62.

7 Romer LM, McConnell AK, Jones DA. Effects of inspiratory muscle training upon recovery time during high intensity, repetitive sprint activity. Int I Sports Med 2002;23:353-60.

8 Spengler CM, Roos M, Laube SM, et al. Decreased exercise blood lactate concentrations after respiratory endurance training in humans. Eur J Appl Physiol Occup Physiol 1999:79:299-305.

9 Stuessi C, Spengler CM, Knopfli-Lenzin C, et al. Respiratory muscle endurance training in humans increases cycling endurance without affecting blood gas concentrations. Eur J Appl Physiol 2001;84:582-6.

10 Volianitis S, McConnell AK, Koutedakis Y, et al. Inspiratory training improves rowing performance. Med Sci Sports Exerc 2001;33:803-9.

11 Fairbarn MS, Coutts KC, Pardy RL, et al. Improved respiratory muscle endurance of highly trained cyclists and the effects on maximal exercise performance. Int I Sports Med 1991;12:66-70.

12 Hanel B, Secher NH. Maximal oxygen uptake and work capacity after inspiratory muscle training: a controlled study. J Sports Sci 1991;9:43-52.

13 Inbar O, Weiner P, Azgad Y, et al. Specific inspiratory muscle training in welltrained endurance athletes. Med Sci Sports Exerc 2000;32:1233-7.

$14 \mathrm{Kohl} J$, Koller EA, Brandenberger $M$, et al. Effect of exercise-induced hyperventilation on airway resistance and cycling endurance. Eur J Appl Physiol Occup Physiol 1997:75:305-11.

15 Morgan DW, Kohrt WM, Bates BJ, et al. Effects of respiratory muscle endurance training on ventilatory and endurance performance of moderately trained cyclists. Int J Sports Med 1987;8:88-93.

16 Williams JS, Wongsathikun J, Boon SM, et al. Inspiratory muscle training fails to improve endurance capacity in athletes. Med Sci Sports Exerc 2002;34: $1194-8$

17 Sonetti DA, Wetter TJ, Pegelow DF, et al. Effects of respiratory muscle training versus placebo on endurance exercise performance. Respir Physiol 2001;127:185-99

18 Quanjer PH, Tammeling GJ, Cotes JE, et al. Lung volumes and forced ventilatory flows. Report Working Party Standardization of Lung Function Tests, European Community for Steel and Coal. Official Statement of the European Respiratory Society. Eur Respir J Suppl 1993;16:5-40.

19 Green M, Road J, Sieck GC, et al. Tests of respiratory muscle strength. ATS/ ERS Statement on Respiratory Muscle Testing. Am J Respir Crit Care Med 2002; 166:518-624

20 Laghi F, D'Alfonso N, Tobin MJ. Pattern of recovery from diaphragmatic fatigue over 24 hours. J Appl Physiol 1995;79:539-46.

21 Chatham K, Baldwin J, Griffiths H, et al. Inspiratory muscle training improves shuttle run performance in healthy subjects. Physiotherapy 1999;85:676-85

22 Ojaunen $M$. Can the true effects of exercise on psychological variables be separated from placebo effects? Int J Sport Psychol 1994:25:63-80.

23 Caine MP, McConnell AK. Pressure threshold inspiratory muscle training improves submaximal cycling performance. Proceedings of the Third Annual Congress of the European College of Sport Science, Manchester, 1998. No 101 .

24 Tzelepis GE, Vega DL, Cohen ME, et al. Lung volume specificity of inspiratory muscle training. J Appl Physiol 1994;77:789-94.

25 Chatwin $M$, Hart N, Nickol AH, et al. Low frequency fatigue induced by a single inspiratory muscle training session [abstract]. Thorax 2001;55:A51.

26 Harms CA, Wetter TJ, St Croix CM, et al. Increased power output at $\mathrm{VO}_{2}$ max with respiratory unloading. Med Sci Sports Exerc 1998;30:S41.

27 Harms CA, Wetter TJ, St Croix CM, et al. Effects of respiratory muscle work on exercise performance. J Appl Physiol 2000;89:131-8.

28 Swanson GD. Pulmonary training may alter exertional dyspnea and fatigue via an exercise-like training effect of a lowered heart rate. Adv Exp Med Biol 1998:450:231-6.

29 Kellerman BA, Martin AD, Davenport PW. Inspiratory strengthening effect on resistive load detection and magnitude estimation. Med Sci Sports Exerc 2000;32:1859-67. 